\title{
Non-Linear Radial Pulsations Driven by Strange Modes in High-Luminosity Helium Stars
}

\author{
P. Montañés Rodríguez, C. S. Jeffery \\ Armagh Observatory, Armagh, Northern Ireland, U.K.
}

\begin{abstract}
We report new non-linear pulsation models for low-mass high-luminosity helium stars. These are compared with the observed periods of radially pulsating extreme helium stars.
\end{abstract}

\section{Introduction}

All hydrogen-deficient high-luminosity stars $(\log L / M>4)$ are believed to pulsate (Saio \& Jeffery, 1988). Observations have identified radial and/or non-radial modes in over seven extreme helium stars (EHes), ranging in effective temperature from $10000-30000 \mathrm{~K}$. The cooler R CrB stars may be related to EHes and show similar behaviour. The periods of both groups are, however, not strictly regular but appear to be chaotic. Linear theory has identified strange-mode excitation as the dominant driving mechanism (Saio \& Wheeler, 1985; Saio \& Jeffery, 1988). The question is whether non-linear theory can explain the observed amplitudes and the irregularity of the periods in EHes.

\section{Models}

We have used a non-linear pulsation code (Bridger, 1984; Christy, 1967; Montañés Rodríguez \& Jeffery, 2001), which includes recent OPAL (Iglesias \& Rogers, 1996) and Alexander (Alexander \& Ferguson, 1994) opacity data, to construct five models of pulsating helium stars. The composition was given by mass fractions $X=0.0, Y=0.98, Z=0.02$, with a standard mixture of metals. The models were chosen to cover a range in mass $\left(0.7-0.9 \mathrm{M}_{\odot}\right)$, luminosity $(11000$ $\left.25000 \mathrm{~L}_{\odot}\right)$ and effective temperature $(10000-18000 \mathrm{~K})$, corresponding to those of radially pulsating EHes.

The models yield fairly irregular pulsations. We performed Fourier analyses of the light curves (Fig. 1). In three cases (models 1, 2 and 4) these are coherent, giving strong single peaks at periods of $14.7,10.8$ and 7.2 days respectively. For comparison, the EHes FQ Aqr, V2244 Oph and PV Tel have periods of approximately 21, 11 and 7 days respectively (Jeffery et al., 2001), but their periods appear to be chaotic.

In two cases (models 3 and 5), the pulsations are more irregular and a single period is harder to identify. This may be a consequence of the extreme non-adiabacity of the pulsations or of the excitation mechanism (strange modes). 

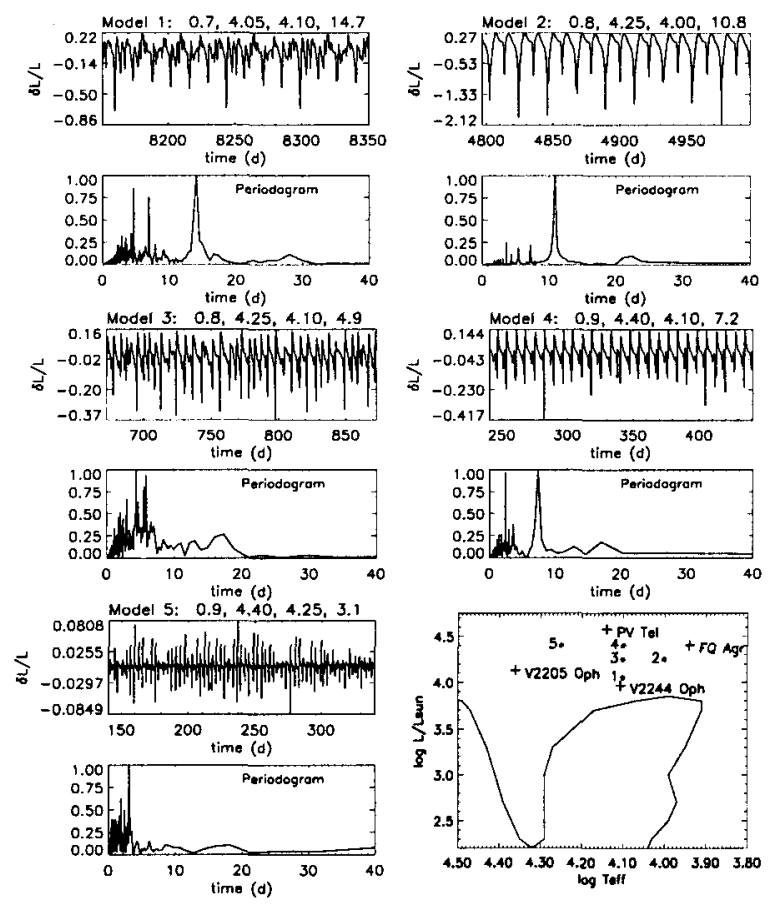

Figure 1. Theoretical luminosity curves and periodograms for models 1-5. Next to the model number: the stellar mass $\left(\mathrm{M}_{\odot}\right), \log \left(\mathrm{L} / \mathrm{L}_{\odot}\right)$, $\log T_{\text {eff }}$ and dominant period (in days). The lower-right panel locates the models and pulsating EHes within the H-R diagram. The instability boundary for $0.7 \mathrm{M}_{\odot}$ and $Z=0.01$ is also shown.

A comparison of the models with observed light and velocity curves for pulsating helium stars and a discussion of role of strange-mode instability in these models will be presented elsewhere.

\section{References}

Alexander, D.R. \& Ferguson, J.W. 1994, ApJ, 437, 879A

Bridger, A. 1984, in Cepheids: Theory \& Observations, (Knudsen), 246

Christy, F. 1966, ARA\&A, 4, 353

Iglesias, C.A. \& Rogers F.J. 1996, ApJ, 464, 943

Jeffery, C.S., Starling, R.L.C., Hill, P.H., \& Pollaco, D. 2001, MNRAS, 321, 111

Montañés Rodríguez, P. \& Jeffery, C.S. 2001, A\&A, submitted

Saio, H. \& Jeffery, C.S. 1988, ApJ, 328, 714

Saio, H. \& Wheeler, J.G. 1985, ApJ, 295, 38 